\title{
CONFOTO: A Semantic Browsing and Annotation Service for Conference Photos
}

\author{
Benjamin Nowack \\ appmosphere web applications, Essen, Germany \\ bnowack@appmosphere.com
}

\begin{abstract}
CONFOTO $^{1}$ is a semantic browsing and annotation service for conference photos. It combines recent Web trends with the advantages of Semantic Web platforms. The service offers several tools to upload, link, browse and annotate pictures. Simple forms can be used to create multilingual titles, tags, or descriptions, while more advanced forms allow the relation of pictures to events, persons, ratings, and copyright information. CONFOTO provides tailored and interlinked browsers for photos, people, events, and documents. Remotely maintained photo descriptions can be added to the local knowledge base, data re-use is made possible via customizable syndication functions and a query interface.
\end{abstract}

\section{Introduction}

CONFOTO is a browsing and annotation service for conference photos. It combines the flexibility of the Resource Description Framework (RDF)[1] with recent Web trends such as keyword-based classification (so-called "folksonomies"[2]), interactive user interfaces[3][4], and syndication of news feeds. The main advantage of utilizing folksonomies is the ease of metadata creation. Online services such as Flickr ${ }^{2}$ were able to attract a large number of users in a relatively short amount of time. However, simple tagging has its limits, as the retrieval of precise or implicit information is not possible. Additionally, a standardized way to directly re-use data does not exist. RDF, on the other hand, is a framework to create fine-grained annotations, but doesn't enjoy a good reputation in terms of simplicity.

CONFOTO is one of the first applications that provides both an end-user-oriented browsing and editing front-end for rich annotations and also a W3C-compliant ${ }^{3}$ interface[5] to an RDF-based data store. It supports the Semantic $\mathrm{Web}^{4}$ idea by allowing resource descriptions to be imported, created, annotated, combined, exported, and re-purposed.

\section{Tools and Features}

CONFOTO uses a set of wrappers to enable photo and conference data import from several different input formats, e.g. RSS 2.0 feeds from w3photo[6], Atom feeds from

\footnotetext{
${ }^{1}$ http://www.confoto.org/

${ }^{2} \mathrm{http}: / /$ flickr.com/

${ }^{3} \mathrm{http} / / / \mathrm{www} . \mathrm{w} 3$. org/2001/sw/DataAccess/

${ }^{4}$ http://www.w3.org/2001/sw
} 
Flickr, or proprietary XML documents from events such ESWC $2005^{5}$ and XTech $2005^{6}$. The system can generate and enhance RDF data for uploaded pictures, for image files linked via Web-accessible URLs, and also for photos described in external RDF/XML[7] documents.

Semantically, CONFOTO is optimized for information about conferences and photos. However, the RDF model allows any resource description to be freely combined with related objects (e.g. a FOAF[8] file or a list of publications could be associated with a person depicted in a photo).

The sections below give an overview of the tools and features currently available at confoto.org.

\subsection{Image Upload or Linking}

Image files can be uploaded via a simple HTML form. A group of uploaded photos can be annotated with associated date, conference, license, copyright, and/or subject information. The system automatically creates image thumbnails and a scaled image for the photo browser.

Another option is to not copy already published pictures to the server but to simply add photo URLs to the local RDF store. This is done through CONFOTO's "Link remote images" form which accepts a list of Web locations and allows groupannotations as well.

For images which have been described in RDF, the service provides an "Add $\mathrm{RDF} / \mathrm{XML}$ " form that can be used to import remotely maintained resource descriptions. Again, thumbnails will be created automatically.

\subsection{Photo Browser}

The photo browser is based on a generic RDF viewer which has been adjusted to generate galleries of clickable thumbnails. Several filters can be used to create custom photo sets. Selecting an image opens a details view which shows a larger version of the image and a list of annotations from the RDF store. In case of non-literal annotations that point to related resources (e.g. persons depicted in the selected photo), the browsers provides a list of labels (e.g. titles of publications, or names of persons) and links to other tools such as a person, event, or document browser when available. This functionality is implemented by utilizing inference capabilities of an underlying OWL[9] toolkit[10].

\subsection{Annotators}

The main difference between CONFOTO and most existing Semantic Web applications is the availability of browser-based annotation forms. Depending on the type of a selected resource, a list of potential relations and attributes is offered to the user. Where possible, the tools support the annotation creation process by interactively suggesting matching resources as shown in Figure 1. This mechanism allows the seamless re-use of data already existing in the RDF store.

\footnotetext{
${ }^{5} \mathrm{http}: / /$ www.eswc2005.org/

${ }^{6} \mathrm{http} / / / \mathrm{www} . x t e c h-c o n f e r e n c e . o r g / 2005 /$
} 


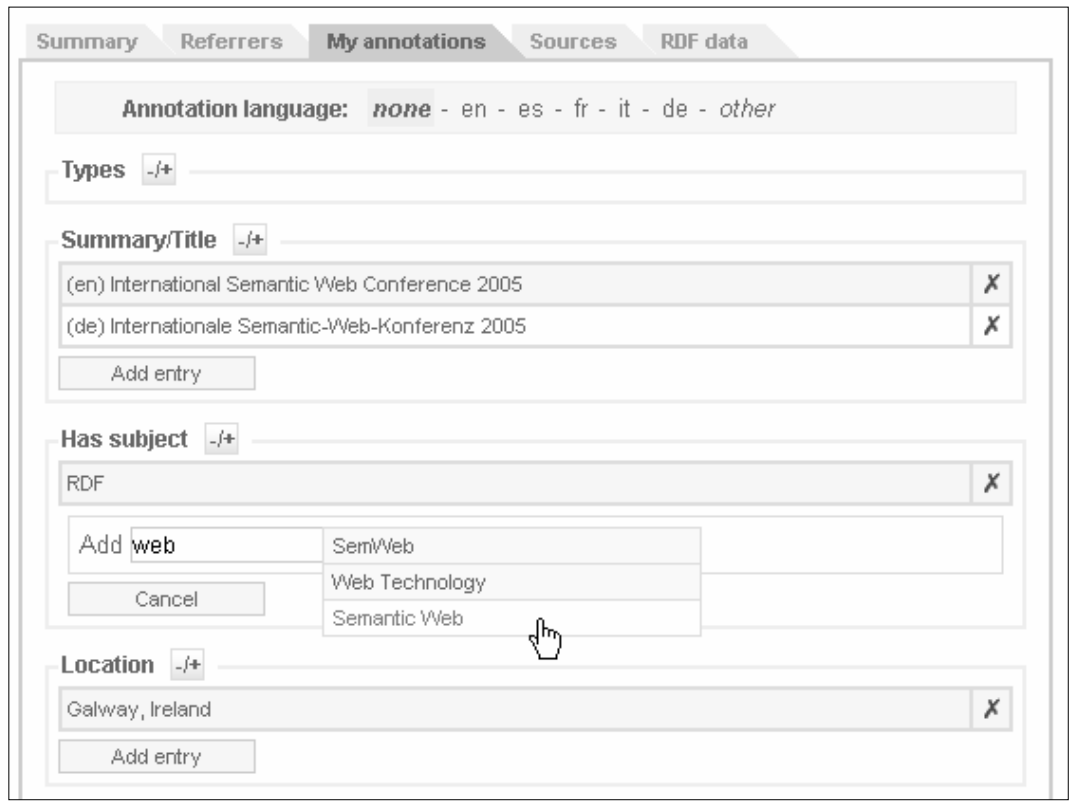

Fig. 1. Annotator with Suggest-as-you-Type Feature

Annotations can directly be added to the RDF store, whereupon the system's inference scripts are executed, iteratively improving the browsing experience. Annotators are rewarded with enhanced results when they switch back to browsing mode.

\subsection{Data Export for Re-use}

CONFOTO features multiple possibilities to export local data: Each page provides a link to an RDF/XML version of the currently displayed resource(s). Apart from that, machine-readable resource descriptions can be obtained by URIQA[11] requests, and also via a basic SPARQL[12] interface. Finally, as the number of URIQA- or SPARQL-enabled tools is still small, custom photo galleries can be exported as simple RSS 1.0[13] news feeds.

\section{Conclusion and Possible Future Work}

CONFOTO demonstrates the advantages of an RDF-based infrastructure and shows, that end-user-oriented, Web-based annotation tools don't have to be limited to simple tagging, but can also be used to create and augment rich annotations. However, the system is still in an early stage and a lot of things could be improved:

To better demonstrate the possibilities of RDF and SPARQL, the browsers need to be extended to offer means for context-specific views such as "all photos taken by this person", or "all events attended by this person". 
CONFOTO maintains provenance information for each annotation. This could be utilized to facilitate navigating the available photos and annotations.

The resource browsers support multiple languages, but the ontologies used are currently only available in English. It is planned to translate the labels of selected terms, so that CONFOTO's browsers can be used in different languages.

\section{References}

1. Resource Description Framework (RDF). W3C (2004). http://www.w3.org/RDF/

2. Folksonomy - Wikipedia. http://en.wikipedia.org/wiki/Folksonomy

3. Remote Scripting with IFRAME. Apple Developer Connection (2002) http://developer. apple.com/internet/webcontent/iframe.html

4. Garrett, J. J. Ajax: A New Approach to Web Applications. (2005) http://www.adaptivepath. com/publications/essays/archives/000385.php

5. Clark, K. G. SPARQL Protocol for RDF. W3C. http://www.w3.org/TR/rdf-sparqlprotocol/

6. w3photo - A Semantic Photo History of the IW3C2 Conferences. http://w3photo.org/

7. Beckett, D. RDF/XML Syntax Specification (Revised). W3C (2004). http://www.w3. org/TR/rdf-syntax-grammar/

8. the friend of a friend (foaf) project http://www.foaf-project.org/

9. Patel-Schneider, P. F., Hayes, P., Horrocks, I. OWL Web Ontology Language Semantics and Abstract Syntax. W3C (2004). http://www.w3.org/TR/owl-semantics/

10. Nowack, B. OWLCHESTRA: Facilitating the Development and Publishing of Small-Scale Web Ontologies. (2004). http://www.appmosphere.com/prod/media/ owlchestra_demo_esws2004.pdf

11. Stickler, P. URIQA: The Nokia URI Query Agent Model. (2003) http://sw.nokia. com/uriqa/URIQA.html

12. Prud'hommeaux, E., Seaborne, A.: SPARQL Query Language for RDF. W3C (2005). http://www.w3.org/TR/rdf-sparql-query/

13. RDF Site Summary (RSS) 1.0. http://web.resource.org/rss/1.0/spec 